\title{
Mechanisms of ischemic skeletal muscle regeneration mediated by mechanically constrained human allogeneic mesenchymal stromal cells
}

\author{
Alex O'Connor ${ }^{1}$, Steven Miller ${ }^{2}$, Michael Loke ${ }^{3}$, Chang-Hyun Gil ${ }^{2}$, Katherin Leckie ${ }^{2}$, Theresa \\ Doiron², Justin King², Leni Moldovan², Michael Murphy² \\ ${ }^{1}$ Indiana University School of Medicine, ${ }^{2}$ Indiana University School of Medicine, Department of \\ Vascular Surgery, ${ }^{3}$ Marian University College of Osteopathic Medicine
}

\begin{abstract}
Background and Hypothesis: Critical limb threatening ischemia (CLTI) is the end stage of peripheral arterial disease (PAD). CLTI presents a significant risk for lower extremity amputation, especially in diabetic patients with poor options for revascularization. Additionally, using a novel diabetic mouse model of CLTI, we have shown that IM injection of 3D cultured MSCs (spheroids) is more effective in promoting skeletal muscle regeneration of ischemic muscle than monolayer cultured MSC. We hypothesize that this result is due to the mechanical constrained nature of the MSC in spheroid form, which has been shown to alter the cellular phenotype. Alginate encapsulated cells are another form of mechanical confinement, and have additional benefits including resistance to immunological attack, making them a practical therapy for treatment of CLTI patients.
\end{abstract}

Project Methods: In this work, cells were encapsulated in $2 \%$ alginate using a centrifugation method. Media from these cells along with others were analyzed for IL-10 and IL-33 using ELISA. Mouse tissue samples were stained with WGA-555 antibody and analyzed using a scanning microscope. Effects on tissue perfusion were measured with Laser Doppler Perfusion Imaging. At the time of this abstract, the media from encapsulated and naked MSCs is being used to culture myoblasts, looking at cell growth.

Results: ELISA results did not show significant increases in IL-10 or IL-33 for encapsulated vs. non-encapsulated cells. LDPI has shown an increased perfusion rate for hindlimbs treated with encapsulated MSCs vs naked MSCs. Muscle fiber analysis is ongoing, but initial data appears promising.

Conclusion and Potential Impact: This experiment provides a starting point for improving and expanding cell therapy for critical limb ischemia, potentially resulting in better outcomes for diabetic patients and preventing lower limb amputations. The encapsulation process also has value in other types of cell therapy, as it could protect cells from host defenses and increase dwell time. 